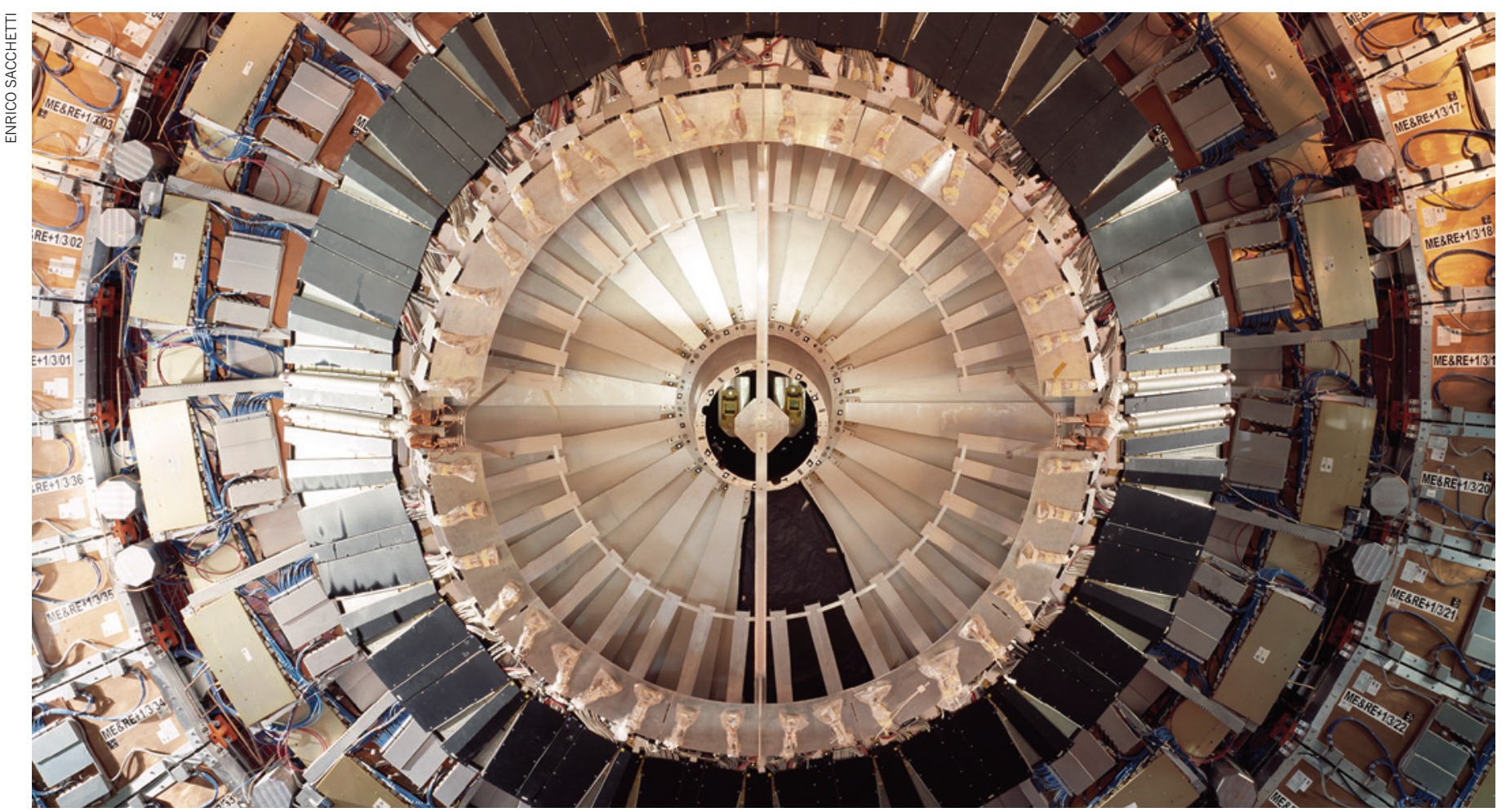

Data collected by experiments at the Large Hadron Collider (including its CMS detector, pictured) have provided little support for supersymmetry theory.

\title{
Truant particles turn the screw on supersymmetry
}

\section{Physicists hoping for signs of radically new particles get nojoy from Large Hadron Collider.}

\section{BY MATTHEW CHALMERS}

A bsence of evidence is not necessarily evidence of absence. That's what theoretical physicist John Ellis of King's College London says of the latest result in the search for supersymmetry, an idea that has captivated particle physicists for 30 years.

Although researchers are still digesting the discovery of the Higgs boson, announced in July at CERN, Europe's particle-physics laboratory near Geneva in Switzerland, more exotic creations are needed to fill out their picture of the subatomic world. The Higgs provides the last missing particle of the standard model of particle physics, but this theory still has some major deficiencies - it is silent on gravity and on what makes up dark matter, among other things. Supersymmetry (SUSY), which predicts that every standard-model particle has a heavier partner, is a step towards a more unified theory of the particles and forces.

Last week at a conference in Kyoto, Japan, physicists working on the LHCb experiment
- one of four large detectors located around CERN's Large Hadron Collider (LHC) announced the hotly anticipated results of an indirect search for new superparticles, known by the cognoscenti as 'sparticles'. The team clocked an extremely rare process in which a $\mathrm{B}_{S}$ meson - composed of a strange quark and a bottom antiquark - decays into a muonantimuon pair. Only one in roughly every 300 million $B_{S}$ mesons is predicted to do this, because the decay relies on a highly unlikely chain of events involving the fleeting appearance of virtual particles. But with the help of sparticles, the rate could increase by perhaps a factor of 100 .

However, the LHCb team found that the $\mathrm{B}_{S}$ behaves just as the standard model says it should, although further data are needed to confirm the measurement. Some members of the 800-strong collaboration proclaimed the result as "very damaging” for SUSY. But SUSY

\section{$\rightarrow$ NATURE.COM}

Read more at

Nature's LHC

special.

www.nature.com/lhc is a slippery animal — it introduces more than 100 new parameters into the standard model, all adjustable - and SUSY proponents don't seem particularly worried. "To paraphrase Mark Twain, news of SUSY's death has been greatly exaggerated," says theoretical physicist and SUSY pioneer Savas Dimopoulos of Stanford University in California.

Nevertheless, the LHC has undoubtedly lengthened the odds of SUSY being correct, at least in its simplest, 'natural' incarnations. "Models of natural supersymmetry are looking increasingly hard to believe," says Brian Feldstein of the Kavli Institute for the Physics and Mathematics of the Universe at the University of Tokyo.

The LHCb result comes on top of tough direct constraints from the LHC's larger ATLAS and CMS detectors. These have seen no sign of sparticles such as squarks and gluinos - nor of any particles outside the standard model. Updates from ATLAS and CMS, presented in Kyoto, continue to show that the lightest superparticles, if they exist, must 
- have truly elephantine masses of more than 1 teraelectronvolt $(\mathrm{TeV})$.

Studies of the Higgs boson itself are yielding mixed signals for SUSY-seekers. So far, rough measurements of CERN's new boson - updated last week - suggest that it behaves just as the standard model predicts, with no hint that additional particles are influencing its behaviour. Yet the mass of the Higgs, 126 gigaelectronvolts $(\mathrm{GeV})$, is pretty much what SUSY predicted, says Sven Heinemeyer at the Institute of Physics of Cantabria in Spain. "If it had been $140 \mathrm{GeV}$ or more, the minimal supersymmetric standard model would have been dead without any possibility of resurrection," he says. SUSY also predicts the existence of four further Higgs particles, at different masses, which have yet to be discovered.

The next chance for some cheering news about SUSY will come in March, when physicists will present results encompassing much more of the data gathered by the LHC, which will continue to smash protons until 17 December. SUSY will probably then remain in limbo until early 2015, when the LHC will re-open for experiments after a long shutdown for maintenance, and will begin colliding protons at energies of $13 \mathrm{TeV}$. So far, the collider has operated at energies of up to only $8 \mathrm{TeV}$. The increase in energy will put discoveries of more massive particles within the LHC's reach.

Yet sparticles with masses of several $\mathrm{TeV}$ or higher might be too heavy to be produced in the LHC or any other conceivable experiment - a troubling possibility.

One of the main attractions of SUSY is that it solves a problem in the standard model, which predicts that the Higgs field - the constant sea of energy from which the Higgs boson arises - should be ramped up to ridiculously high values by the everpresent fluctuations of standard-model particles. SUSY's sparticles are supposed to prevent that by cancelling out the fluctuations, thereby stabilizing the Higgs. But there is disagreement about whether superheavy sparticles can offer enough stabilization.

In another blow to SUSY's explanatory power, such sparticles could also become too heavy to be convincing candidates for dark matter, the unseen stuff that astronomers believe has a key role in shaping cosmic structure.

Fans of SUSY are likely to lose faith only if the LHC finds nothing after a year or two of high-energy running. That would force them to look for an even more audacious idea to break free from the standard model. Even so, SUSY will probably live on in mathematical physics, says theorist Ben Allanach of the University of Cambridge, UK. "It is such a powerful new mathematical symmetry that it would be a shame if nature didn't make use of it," he says. .

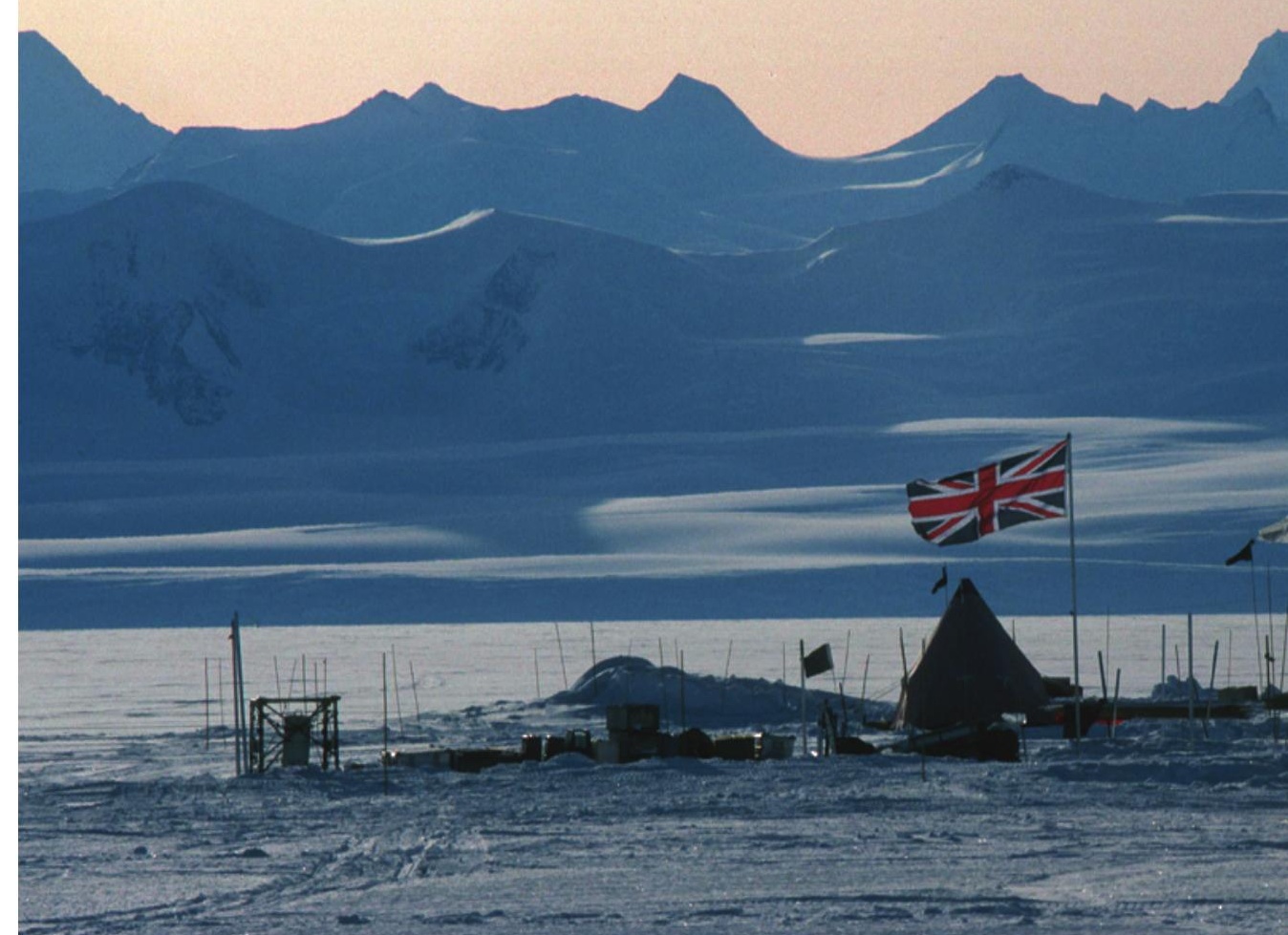

A British field camp in Antarctica will soon host efforts to drill through the ice to reach Lake Ellsworth.

\section{EARTH SCIENCE}

\section{Hunt for life under Antarctic ice heats up}

\section{UK and US teams to drill into ancient subglacial lakes.}

\section{BY QUIRIN SCHIERMEIER}

$\mathrm{N}$ estled in a steep fjord beneath three kilometres of Antarctic ice, the lost world of Lake Ellsworth has haunted Martin Siegert's dreams ever since he got involved in subglacial research a dozen years ago. Finally, the time has come for him to explore its mysterious waters.

Next week, Siegert, a glaciologist at the University of Bristol, UK, packs his bags for the long journey to the opposite end of the world. Once he has reached the Rothera Research Station of the British Antarctic Survey (BAS) on an island off the Antarctic Peninsula, he and his science crew will fly about 1,000 kilometres into western Antarctica. On 5 December, the real work begins: drilling straight down through the ice to the pristine lake beneath. In its shadowy waters they hope to find forms of life that have not seen the light of day in millions of years (see 'Trapped under ice'). And in the lake bed sediments, the team will search for records of the poorly understood history of the West Antarctic Ice Sheet, potentially revealing how the mighty glacier has waxed and waned over time.

Is Siegert excited? "This is the very pinnacle of the science I've been doing since the turn of the millennium," he says. "Now guess if I'm excited."

Almost 380 subglacial lakes have been discovered and mapped in Antarctica, and have been explored remotely with ice-penetrating radar, gravity measurements and seismic investigations (A. Wright and M. Siegert Antarctic Science http://doi.org/jsn; 2012). These ancient lakes, large and small, owe their existence to geothermal heat that melts the Antarctic ice from below. Gravity and ice pressure force the melt water to flow, and it collects in the hollows and valleys of the continent under the ice.

If all goes to plan, Lake Ellsworth will be the second such lake to be breached. In February, a Russian team penetrated Lake Vostok - the
ONATURE.COM Read more about the exploration of Lake Vostok: go.nature.com/h55xgu largest and deepest Antarctic lake - completing a project that was launched more than 20 years ago (see Nature 482, 287; 2012). And 